\title{
Piecewise Straight Line Approximation of Curve Existing in Slightly Curved Mesiobuccal Root Canal of Mandibular First Molar. A Radiographic Investigation
}

\author{
Aproximación a Segmentos de Línea Recta en la Curva Existente del Canal Radicular \\ Mesiobucal Ligeramente Curvado del Primer Molar Inferior. Una Investigación Radiográfica
}

Prabhakar, J.*; Priya, M. S..*; L. Jones Tarcius Doss**** \& Sukumaran, V. G.****

PRABHAKAR, J.; PRIYA, M. S.; DOSS, L. J. T. \& SUKUMARAN, V. G. Piecewise straight line approximation of curve existing in slightly curved mesiobuccal root canal of mandibular first molar. A radiographic investigation. Int. J. Morphol., 31(1):131-135, 2013.

SUMMARY: The aim of this study was to radiographically investigate the curve existing in slightly curved mesiobuccal canal of mandibular first molar by applying Piecewise straight line approximation. Extracted human mandibular molars were radiographed and one hundred radiographs were selected whose mesiobuccal canal showed slight curvature (10-20 $)$ according to Schneider's method. The curves were traced and analyzed using Piecewise straight line method. Each curve was considered as a unit consisting of six different pieces of straight lines joining at seven specific points and the angle of curvature at these points was determined using the slope formula. All curves analyzed in this study had varying degrees of curvatures at different points on the curve. Maximum curvature $\left(0.40^{\circ}\right)$ was recorded at the middle third of the root canal. Within the limitation of the study, significant curvature occurs through out the curve existing in the mesiobuccal canal of mandibular first molar and the middle third of the curve showing greater degree of curvature. There is a possibility of greater curvature occurring in the coronal third of the curve. Though three dimensional studies would be more appropriate, Piecewise straight line approximation may be a better method than existing methods to simulate canal geometry.

KEY WORDS: Piecewise straight lines; Canal curvature; Mesiobuccal canal; Schneider method; Curvature radius; Canal simulation; Angle of curvature.

\section{INTRODUCTION}

Clinical success of any root canal therapy is enhanced with thorough knowledge of root canal morphology (Baisden et al., 1992). Biomechanical preparation of curved root canals is challenging, as different degrees of root curvature lead to different difficulties in canal preparation. Undetermined canal morphology increases the risk of transportation, ledge formation, and even perforation (Mullaney, 1979; Weine et al., 1976; Roane et al., 1985). The difficulties in preparation of curved root canals have prompted the development of new preparation methods, instruments and investigation of root canal geometry (Hankins \& ElDeeb, 1996; Kyomen et al., 1994; Esposito \& Cunningham, 1995; Pruett et al., 1997; Harlan et al., 1996). The degree of curvature of root canal is considered to be one of the important parameter that influences the accuracy of preparation and separation of instruments in the root canal (Zelada et al., 2002).
Schneider (1971) described the most easily applicable method to determine the degree of curvature in root canals. He divided root canal curvatures into straight root canals $\left(<5^{\circ}\right)$, slightly curved canals $\left(10-20^{\circ}\right)$ and severely curved canals $\left(25-70^{\circ}\right)$. Bone \& Moule (1986) described secondary curvature in the apical region by modifying Schneider's method and other alternate methods were proposed by Weine (1976), Hankins \& ElDebb. Pruett et al., introduced a new parameter described as the "curvature radius" for measuring canal curvature.

Piecewise straight line approximation is a connected series of line pieces, in which a curve is specified by a sequence of points $(\mathrm{x}, \mathrm{y})$ called coordinates so that the curve consists of the line pieces connecting the consecutive coordinates. A finite set of points (xi,yi) for $\mathrm{i}=0,1,2, \ldots, \mathrm{n}$ (called "nodal points") on the curve is selected. The curve is

* Department of Conservative Dentistry, Tagore Dental College \& Hospital, Chennai, India.

** Department of Periodontics, Tagore Dental College \& Hospital, Chennai, India.

${ }^{* * *}$ Assistant Professor, Department of Mathematics. Anna University, Chennai, India.

***** Department of Conservative Dentistry, Sree Balaji Dental College \& Hospital, Chennai, India. 
then approximated through piecewise straight lines by joining consecutive nodal points (xi, yi) and (xi+1, yi+1) for $\mathrm{i}=0,1,2, \ldots, \mathrm{n}-1$ lying on the curve. By varying the value of $\mathrm{n}$ i.e., by selecting more nodal points, we obtain a better approximation of the curve.

The aim of this study was to radiographically investigate the curve existing in slightly curved mesiobuccal canal of mandibular first molar by applying Piecewise straight line approximation.

\section{MATERIAL AND METHOD}

Extracted human mandibular first molars were collected. Selection criteria eliminated teeth with previous root canal therapy, incompletely formed apices, fractured roots, external resorption and narrow or obstructed canals. Coronal access was made using diamond burs. Without prior instrumentation of the root canal, a size $08 \mathrm{~K}$ type reamer (Dentsply Maillefer, Balaigues, Switzerland) was inserted into the mesiobuccal canal and gently advanced until it was visible at the apical foramen.

The tooth was attached to Kodak Ultra speed film (Kodak, Stuttgart, Germany) with soft wax and was aligned so that the long axis of the root canal was parallel, and as near as possible to the surface of the $\mathrm{x}$-ray film. The $\mathrm{x}$-ray tube was aligned perpendicular to the root canal and the radiographs were made in clinical view.

The exposure time was the same for all radiographs with a constant source to film distance of $50 \mathrm{~cm}$ and an object to film length of $5 \mathrm{~mm}$. Then, the films were developed, fixed and dried. The proximal view of the root canal in mesiodistal direction was also made. The canals with secondary curvature were not included in this study. One hundred radiographs were selected whose mesiobuccal canal showed slight curvature (10$20^{\circ}$ ) according to Schneider's method.

The selected radiographs were scanned and curves were traced under 30X magnification using Curve Tracing Software (College of Engineering, Chennai, India). One hundred independent root canal curves were analyzed using Piecewise straight line method. In this method, the whole length of the canal was measured along the $\mathrm{Y}$ axis from the apex to the orifice and divided into six equal parts by selecting seven equidistant points. The $(x, y)$ coordinates of each curve were plotted. The point $(\mathrm{x} 0, \mathrm{y} 0)$ corresponds to the nodal point at apex and $(\mathrm{x} 6, \mathrm{y} 6)$ corresponds to the nodal point at orifice whereas the remaining points correspond to points in between. Seven $(\mathrm{x}, \mathrm{y})$ coordinates were recorded for each curve from apex to the orifice as $(\mathrm{x} 0, \mathrm{y} 0),(\mathrm{x} 1, \mathrm{y} 1)(\mathrm{x} 2, \mathrm{y} 2)(\mathrm{x} 3, \mathrm{y} 3)(\mathrm{x} 4, \mathrm{y} 4)$ (x5,y5) (x6,y6) (Fig. 1a).

Each curve was considered as a unit consisting of six different pieces of straight lines joining at seven nodal points. Each curve is divided into three parts namely coronal, middle and apical segment with each segment consisting of two pieces of straight line.

The angle of curvature at an intermediate nodal point (xi, yi) for $\mathrm{i}=1,2, \ldots 5$ can be approximately evaluated by computing the angle between the two straight line pieces namely the line joining (xi-1,yi-1) and (xi,yi) and the line joining (xi,yi) and $(x i+1, y i+1)$. Now the angle between these two straight lines can be computed using the slope formula as follows:

Let $\mathrm{m} 1$ be the slope of the line joining (xi-1,yi-1) and (xi,yi) and $\mathrm{m} 2$ be that of the line joining (xi,yi) and $(x i+1, y i+1)$. Then the angle $q$ is given by the formula

$$
\tan \mathrm{q}=\frac{(\mathrm{m} 1-\mathrm{m} 2)}{(1+\mathrm{m} 1 \mathrm{~m} 2)}
$$

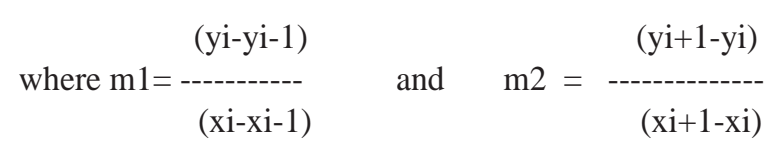

Five q values were obtained for each curve, two each for apical and middle segments and one for the coronal segment. These $\mathrm{q}$ values were obtained at the starting point and midpoint for apical and middle segment, whereas for the coronal segment q was obtained only at the mid point (Fig 1b).

\section{RESULTS}

All curves analyzed in our study had varying degrees of curvatures at different points on the curve. Table I shows the median and range of curvature existing at five nodal points. The range of $q$ values recorded for points on apical segment $\left(-0.02^{\circ}\right.$ to $-0.11^{\circ}$ and $-0.05^{\circ}$ to $-0.11^{\circ}$ ) was less compared to the points on middle and coronal segment. The maximum curvature $\left(0.40^{\circ}\right)$ was recorded at mid point and starting point of the middle segment. $47 \%$ of $q$ values in the midpoint of middle segment were in the range of $-0.08^{\circ}$ to $-0.19^{\circ}$ whereas $53 \%$ of the values were in the range of $0.13^{\circ}$ to $0.40^{\circ}$. A wide range of $q$ values were recorded in the start point of middle segment $\left(0.07^{\circ}\right.$ to $\left.0.40^{\circ}\right)$ but $68 \%$ of the reading were only in the range of $0.32^{\circ}$ to $0.40^{\circ}$. The range of $\mathrm{q}$ values recorded for mid point of coronal segment is $0.04^{\circ}$ to $0.34^{\circ}$ but only $9 \%$ of values were recorded between $0.32^{\circ}$ and $0.34^{\circ}$, whereas $91 \%$ of values ranged between $0.04^{\circ}$ and $0.11^{\circ}$. 

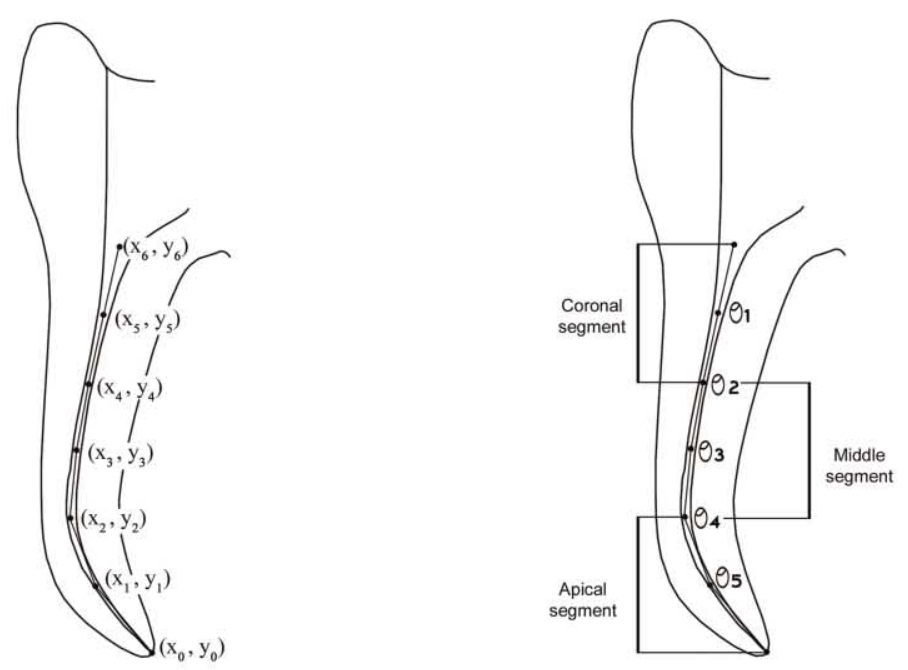

a

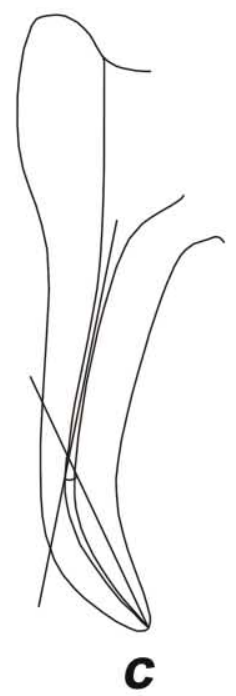

Fig. 1. (a) Seven nodal points with $\mathrm{x}$ and y coordinates. (b) Piecewise straight line approximation with angle of curvatures between pieces.

(c) Schneider method (d) Pruett et al., method.

\section{DISCUSSION}

The most common method of describing a curve is to give its parameterization. Another way, however, is to say how much the curve "bends" at each point. This measure of bending is known by the technical word "curvature". Curvature is all that is needed to define a curve. A curve that has constant curvature must be part or all of a circle (for these are the only curves that have the same amount of bending at every point).

A reamer was used in this study to mark out the mesiobuccal canal instead of traditional $\mathrm{K}$ file or silver cones. The reamer has an advantage when used to copy the canal curvatures, because the flutes are not twisted as the $\mathrm{K}$ file, has less memory and is more adaptable to a three dimensional shape (Jerome \& Hanlon, 2003).

In our study, curvatures existing at the apical third of the analyzed root canals showed a minimal range with lesser q values. The occurrence of $9 \%$ of values between $0.32^{\circ}$ and $0.34^{\circ}$ in the mid point of the coronal segment, presents a possibility of having greater curvatures at the coronal third. This finding enhances the importance of straight line access and preflaring. Günday et al., (2005) showed that canal access angles of two canals with different canal geometry can differ, even if they have the same canal curvature as measured using the Schneider angles. The maximum curvature occurred at the middle segment of the curve and the most frequent was the starting point of the middle segment.

Simulated canals in transparent resin blocks are widely used for standardized evaluation of the cutting characteristics, canal preparation and fracture of endodontic instruments. A variety of

Table I. Angle of curvature at nodal points.

\begin{tabular}{lcccc}
\hline & & \multicolumn{3}{c}{ Angle of curvature in degree } \\
\cline { 3 - 5 } & Curvature at points joining the pieces & Median & Minimum & Maximum \\
\hline 1 & Mid point of Apical Segment & -0.04 & -0.02 & -0.11 \\
2 & Start point of Apical Segment & -0.08 & -0.05 & -0.11 \\
3 & Mid point of Middle Segment & 0.13 & -0.18 & 0.4 \\
4 & Start point of Middle Segment & 0.32 & 0.07 & 0.4 \\
5 & Mid point of Coronal Segment & 0.09 & 0.04 & 0.34 \\
\hline
\end{tabular}


simulated canals are available commercially based on different methods of describing canal curvature and the most commonly available are based on Schneider and Pruett et al., methods.

Schneider employed a method in which a curved canal was expressed with an arbitrary angle of curvature as the only parameter. According to this method (Fig. 1c), canal curvature was determined after drawing a straight line along the longitudinal axis of the root canal in the coronal third and a second straight line from the apex to the point where canal deviates from the longitudinal axis of the tooth. This method gives the angle of deflection between the two intersecting lines. But by definition, each point on a curve has a definite curvature to the adjacent point and it is important to consider the angle of curvature all along the curve. Schneider's pioneering work on canal curvature was a useful tool to clinicians to appreciate the severity of the curvature to be negotiated, but applying it for dental education and research is questionable.

Pruett et al., modified Schneider's method by adding radius as another parameter (Fig 1d). Pruett et al., stated that "two canals measured at the same angle in degrees by the Schneider method could have very different radii or abruptness of curvatures, thus having a very different impact on the difficulty of canal instrumentation. But, only a circle or part of a circle (Arc) can have a radius and they have a constant curvature at each point of the curve, however the curves of the canal are not an arc. Considering the curve existing in the canal as an arc may help us in approximating the curve but the basic idea of canal simulation is compromised as we end up in constant canal curvature throughout the curve.
Piecewise straight line approximation of the curves existing in root canals provides a better simulation as it incorporates more angle of curvatures all along the curve. This method may be helpful in good understanding of the curves in root canals. It may also be useful in evaluating the cutting characteristics, canal preparation and fracture of endodontic instruments.

\section{CONCLUSION}

Within the limitation of the study, significant curvature occurs through out the curve existing in the mesiobuccal canal of mandibular first molar and the middle third of the curve showing a greater degree of curvature. There is a possibility of greater curvature occurring in the coronal third of the curve. Though three dimensional studies would be more appropriate, Piecewise straight line approximation may be a better method than existing methods to simulate canal geometry.

\section{ACKNOWLEDGEMENT}

I affirm that I/We have no financial affiliation (e.g., employment, direct payment, stock holdings, retainers, consultantships, patent licensing arrangements or honoraria), or involvement with any commercial organization with direct financial interest in the subject or materials discussed in this manuscript, nor have any such arrangements existed in the past three years. Any other potential conflict of interest is disclosed.

PRABHAKAR, J.; PRIYA, M. S.; DOSS, L. J. T. \& SUKUMARAN, V. G. Aproximación a segmentos de línea recta en la curva existente del canal radicular mesiobucal ligeramente curvado del primer molar inferior. Una investigación radiográfica. Int. J. Morphol., 31(1):131-135, 2013

RESUMEN: El objetivo de este estudio fue investigar radiográficamente la curva existente en el canal radicular mesiobucal ligeramente curvado del primer molar inferior mediante la aplicación de aproximaciones a segmentos de línea recta. Se utilizaron molares inferiores humanos extraídos que fueron radiografiados. Fueron seleccionadas, según el método de Schneider, 100 radiografías cuyo canal mesiobucal mostró una ligera curvatura $\left(10-20^{\circ}\right)$. Las curvas fueron delineadas y analizadas mediante el método de aproximación a segmentos de línea recta. Cada curva se consideró como una unidad que consta de seis diferentes segmentos de línea recta que unen a siete puntos específicos y el ángulo de curvatura en estos puntos se determinó utilizando la fórmula de la pendiente. Todas las curvas analizadas en este estudio tenían diversos grados de curvaturas en diferentes puntos de la curva. La curvatura máxima $\left(0,40^{\circ}\right)$ se registró en el tercio medio del canal radicular. Dentro de la limitación del estudio, una curvatura significativa se produce a través de la curva existente en el canal mesiobucal del primer molar inferior y el tercio medio de la curva que muestra un mayor grado de curvatura. Existe la posibilidad que ocurra una mayor curvatura en el tercio coronal de la curva. Aunque un estudio tridimensional sería más apropiado, la aproximación a segmentos de línea recta puede ser un mejor método que los existentes para simular la geometría del canal.

PALABRAS ClAVE: Aproximación a segmentos de línea recta; Curvatura del canal; Canal mesiobucal; Método de Schneider; Radio de curvatura; Simulación del canal; Ángulo de curvatura. 


\section{REFERENCES}

Baisden, M. K.; Kulild, J. C. \& Weller, R. N. Root canal configuration of the mandibular first premolar. J. Endod., 18(10):505-8, 1992.

Bone, J. \& Moule, A. J. The nature of curvature of palatal canals in maxillary molar teeth. Int. Endod. J., 19(4):178-86, 1986.

Esposito, P. T. \& Cunningham, C. J. A comparison of canal preparation with nickel-titanium and stainless steel instruments. J. Endod., 21(4):173-6, 1995.

Günday, M.; Sazak, H. \& Garip, Y. A comparative study of three different root canal curvature measurement techniques and measuring the canal access angle in curved canals. J. Endod., 31(11):796-8, 2005.

Hankins, P. J. \& ElDeeb, M. E. An evaluation of the Canal Master, balanced force, and step-back techniques. J. Endod., 22(3):12330, 1996.

Harlan, A. L.; Nicholls, J. I. \& Steiner, J. C. A comparison of curved canal instrumentation using nickel-titanium or stainless steel files with the balanced-force technique. J. Endod., 22(8):410-3, 1996.

Jerome, C. E. \& Hanlon, R. J. Jr. Identifying multiplanar root canal curvatures using stainless-steel instruments. J. Endod., 29(5):356-8, 2003.

Kyomen, S. M.; Caputo, A. A. \& White, S. N. Critical analysis of the balanced force technique in endodontics. J. Endod., 20(7):332-7, 1994.

Mullaney, T. P. Instrumentation of finely curved canals. Dent. Clin. North Am., 23(4):575-92, 1979.

Pruett, J. P.; Clement, D. J. \& Carnes, D. L. Jr. Cyclic fatigue testing of nickel-titanium endodontic instruments. J. Endod., 23(2):7785, 1997.
Roane, J. B.; Sabala, C. L. \& Duncanson, M. G. Jr. The "balanced force" concept for instrumentation of curved canals. J. Endod., 11(5):203-11, 1985.

Schneider, S. W. A comparison of canal preparations in straight and curved root canals. Oral Surg. Oral Med. Oral Pathol., 32(2):271-5, 1971.

Weine, F. S.; Kelly, R. F. \& Bray, K. E. Effect of preparation with endodontic hand piece on original canal shape. J. Endod., 2(10):298-303, 1976.

Zelada, G.; Varela, P.; Martín, B.; Bahíllo, J. G.; Magán, F. \& Ahn, $\mathrm{S}$. The effect of rotational speed and the curvature of root canals on the breakage of rotary endodontic instruments. J. Endod., 28(7):540-2, 2002.

Correspondence to:

Dr. J. Prabhakar, M.D.S

Tagore Dental College \& Hospital

Rathinamangalam, Chennai-600048

INDIA

Phone Number: 91-9840875275

Email: drpriyaprabhakar@gmail.com

Received: $15-03-2012$

Accepted: 19-08-2012 